PROCEEDINGS OF THE

AMERICAN MATHEMATICAL SOCIETY

Volume 133, Number 10, Pages 2911-2920

S 0002-9939(05)07884-6

Article electronically published on May 2, 2005

\title{
COMPACT ORTHOALGEBRAS
}

\author{
ALEXANDER WILCE
}

(Communicated by Lance W. Small)

\begin{abstract}
We initiate a study of topological orthoalgebras (TOAs), concentrating on the compact case. Examples of TOAs include topological orthomodular lattices, and also the projection lattice of a Hilbert space. As the latter example illustrates, a lattice-ordered TOA need not be a topological lattice. However, we show that a compact Boolean TOA is a topological Boolean algebra. Using this, we prove that any compact regular TOA is atomistic, and has a compact center. We prove also that any compact TOA with isolated 0 is of finite height. We then focus on stably ordered TOAs: those in which the upper set generated by an open set is open. These include both topological orthomodular lattices and interval orthoalgebras - in particular, projection lattices. We show that the topology of a compact stably-ordered TOA with isolated 0 is determined by that of its space of atoms.
\end{abstract}

\section{INTRODUCTION}

Broadly speaking, a quantum logic is any of a range of order-theoretic and partialalgebraic structures - orthomodular lattices and posets, orthoalgebras, and effect algebras - abstracted from the projection lattice $L(\mathbf{H})$ of a Hilbert space $\mathbf{H}$. Since the primordial example is very much a topological object, it would seem natural to undertake a study of "topological quantum logics" more generally. There does exist a literature devoted to topological orthomodular lattices (e.g., [3, 4, 12]); however $L(\mathbf{H})$, in its norm or strong operator topology, is not a topological lattice, the meet and join in $L(\mathbf{H})$ being not continuous. On the other hand, $L(\mathbf{H})$ is a topological orthoalgebra in a natural sense - as, indeed, are many other orthoalgebras one meets in practice, including all topological orthomodular lattices.

The purpose of this paper is to begin a systematic study of topological orthoalgebras (TOAs) in abstracto. In the interest of making what follows self-contained, section 2 collects some general background material on orthoalgebras. Section 3 develops some of the general theory of TOAs, with a focus on the compact case. Among other things, it is shown that a compact Boolean TOA is a topological Boolean algebra. This is a non-trivial fact, since, as the example of $L(\mathbf{H})$ shows, a lattice-ordered TOA need not be a topological lattice. We also show that any (algebraically) regular compact topological orthomodular poset is atomistic, and that a compact TOA with isolated zero is atomistic and of finite height. In section 4, we

Received by the editors August 22, 2003 and, in revised form, June 10, 2004.

2000 Mathematics Subject Classification. Primary 06F15, 06F30; Secondary 03G12, 81P10.

Key words and phrases. Orthoalgebra, effect algebra, orthomodular lattice, topological lattice, quantum logic.

(C)2005 American Mathematical Society Reverts to public domain 28 years from publication 
consider a class of TOAs that we call stably ordered: those in which the upper set generated by an open set is again open. This includes all topological orthomodular lattices, and also projection lattices. We show that the topology of a stably-ordered TOA with isolated zero is entirely determined by that on its space of atoms.

\section{BACKGROUND}

If $\left(L, \leq{ }^{\prime}, 0,1\right)$ is any orthocomplemented poset, we call elements $a$ and $b$ of $L$ orthogonal, writing $a \perp b$, iff $a \leq b^{\prime}$. Suppose that any two orthogonal elements of $L$ have a join. Then for $a \leq b$ in $L$, we can define a relative complement $b \wedge a^{\prime} \perp a$. $L$ is an orthomodular poset (hereafter: OMP) iff, in addition,

$$
a \leq b \Rightarrow\left(b \wedge a^{\prime}\right) \vee a=b .
$$

An orthomodular lattice (OML) is a lattice-ordered OMP. Evidently, the "orthomodular identity" (2.1) is a weak form of distributivity, and thus every Boolean algebra is an OML. The primordial (non-Boolean) example is the lattice $L(\mathbf{H})$ of projections - equivalently, closed subspaces - of a Hilbert space $\mathbf{H}$. Orthomodular lattices and posets have been studied extensively. The standard reference is [9]; for a more recent survey, see [2].

Let us agree to write $a \oplus b$ for the join of orthogonal elements $a$ and $b$ of an orthocomplemented poset, whenever this join exists. It is not difficult to check that $L$ is an OMP iff the resulting structure $(L, \oplus)$ satisfies the conditions that (i) $a \oplus b$ exists whenever $a \perp b$, and (ii) if $a \oplus b=1$, then $b=a^{\prime}$. This suggests the following.

Definition 2.1. An orthoalgebra is a structure $(L, \oplus)$ consisting of a set $L$ and an associative, commutative 1 partial binary operation $\oplus$ on $L$, such that for all $a \in L$

(a) there exists a unique element $a^{\prime} \in L$ with $a \oplus a^{\prime}=1$;

(b) $a \oplus a$ exists only if $a=1^{\prime}$.

We write $a \perp b$ to indicate that $a \oplus b$ exists. Also, we write 0 for $1^{\prime}$, noticing that $0 \oplus a=a$ for every $a \in L$. A set $S \subseteq L$ is a sub-orthoalgebra of $L$ iff it contains 0 and is closed under both ' and $\oplus$ (whenever the latter is defined).

Orthoalgebras were introduced in the early 1980 s by D. J. Foulis and C. H. Randall [6] in connection with the problem of defining tensor products of quantum logics. Further information can be found in [5] and [14]. For later reference, we mention that an effect algebra (see, e.g., [1]) is a structure $(L, \oplus)$, satisfying all of the conditions of Definition 2.1 save (b), which is replaced by the weaker condition that for all $a \in L$, if $a \perp 1$, then $a=0$.

2.1. Orthoalgebras as orthoposets. From the remarks preceding Definition 1.1, it is clear that any OMP gives rise to an orthoalgebra in which $a \oplus b=a \vee b$. Any orthoalgebra $(L, \oplus)$ can be partially ordered by setting $a \leq b$ iff there exists $c \in L$ with $b=a \oplus c$. The operation $a \mapsto a^{\prime}$ is an orthocomplementation with respect to this ordering. Thus, any orthoalgebra gives rise to an orthoposet. Moreover, for any $a \leq b$ in $L$, there is a unique element $c \in L$ - namely, $\left(b^{\prime} \oplus a\right)^{\prime}$ - such that $b=a \oplus c$. It is usual to call this element $b \ominus a$. If $L$ is an OMP, this is exactly $b \wedge a^{\prime}$. In this language, the orthomodular law (1) becomes

$$
a \leq b \Rightarrow b=(b \ominus a) \oplus a,
$$

\footnotetext{
${ }^{1}$ The associativity and commutativity of $\oplus$ are here to be understood in the strong sense, i.e., if $a \oplus b$ is defined, then so is $b \oplus a$, and the two are equal, and if $a \oplus(b \oplus c)$ is defined, so is $(a \oplus b) \oplus c$, and the two are equal.
} 
which holds in any orthoalgebra. In general, however, $a \oplus b$ is not the join, but only a minimal upper bound, for orthogonal elements $a$ and $b$ of an orthoalgebra $L$. Indeed, one can show that the orthoposet $\left(L, \leq,,^{\prime}, 0,1\right)$ obtained from $(L, \oplus)$ is an OMP if and only if $a \oplus b=a \vee b$ for all $a, b \in L$; this in turn is equivalent to the condition, called orthocoherence in the literature, that if $a, b, c \in L$ are pairwise orthogonal, then $a \perp(b \oplus c)$, so that $a \oplus(b \oplus c)$ exists. Thus, orthomodular posets are effectively the same things as orthocoherent orthoalgebras, and orthomodular lattices are effectively the same things as lattice-ordered orthoalgebras.

2.2. Boolean orthoalgebras and compatibility. An orthoalgebra $(L, \oplus)$ is said to be Boolean iff the corresponding orthoposet $\left(L, \leq,,^{\prime}, 0,1\right)$ is a Boolean lattice. A subset of $L$ is said to be compatible iff it is contained in a Boolean sub-orthoalgebra of $L$. Two elements $a, b \in L$ are compatible iff there exist elements $a_{1}, b_{1}$ and $c$ with $a=a_{1} \oplus c, b=c \oplus b_{1}$, and $a \perp b_{1}$, so that $a_{1} \oplus c \oplus b_{1}$ exists [5]. Equivalently, $a$ and $b$ are compatible iff there exists an element $c \leq a, b$ with $a \perp(b \ominus c)$. The triple $\left(a_{1}, c, b_{1}\right)=(a \ominus c, c, b \ominus c)$ is then called a Mackey decomposition for $a$ and $b$. If $L$ is Boolean, then every pair of elements $a, b \in L$ has a unique Mackey decomposition, namely, $(a \ominus b, a \wedge b, b \ominus a)$. It is possible, even in an OMP, for a pairwise compatible set of elements not to be compatible. An orthoalgebra in which pairwise compatible sets are compatible is said to be regular. Note that such an orthoalgebra is automatically orthocoherent, i.e., an OMP.

2.3. The center of an orthoalgebra. For any element $a$ of an orthoalgebra $L$, the interval $[0, a]=\{x \in L \mid 0 \leq x \leq a\}$ is itself an orthoalgebra, the orthogonal sum of $x, y \leq a$ being given by $x \oplus y$, provided this exists in $L$ and is again below $a$. There is a natural mapping $[0, a] \times\left[0, a^{\prime}\right] \rightarrow L$ given by $(x, y) \mapsto x \oplus y$. If this is an isomorphism, $a$ is said to be central. The center of $L$ is the set $\mathbf{C}(L)$ of all central elements of $L$. It can be shown $[8]$ that $\mathbf{C}(L)$ is a Boolean sub-orthoalgebra of $L$. In particular, $L$ is Boolean iff $L=\mathbf{C}(L)$. $L$ is irreducible iff $\mathbf{C}(L)=\{0,1\}$.

2.4. Joint orthogonality. A compatible pairwise-orthogonal set is said to be jointly orthogonal. Equivalently, $A \subseteq L$ is jointly orthogonal iff, for every finite subset $F=\left\{a_{1}, \ldots, a_{n}\right\} \subseteq A$, the "partial sum" $\bigoplus F=a_{1} \oplus \cdots \oplus a_{n}$ exists. If the join of all partial sums of $A$ exists, we denote it by $\bigoplus A$ and speak of this as the sum of $A$. We shall say that $L$ is orthocomplete if every jointly orthogonal subset of $L$ has a sum in this sense. An orthoalgebra is atomistic iff every element of $L$ can be expressed as the sum of a jointly orthogonal set of atoms.

\section{TOPOLOGICAL ORTHOALGEBRAS}

Definition 3.1. A topological orthoalgebra (hereafter: TOA) is an orthoalgebra $(L, \oplus)$ equipped with a topology making the relation $\perp \subseteq L \times L$ closed, and the mappings $\oplus: \perp \rightarrow L$ and $^{\prime}: L \rightarrow L$, continuous.

One could define a topological effect algebra in just the same way 2 We shall not pursue this further, except to note that the following lemma would carry over verbatim to that context.

\footnotetext{
${ }^{2}$ In a recent preprint [13], Riečanova offers a definition of topological effect algebras not requiring the relation $\perp$ to be closed.
} 
Lemma 3.2. Let $(L, \oplus)$ be a topological orthoalgebra. Then

(a) the order relation $\leq$ is closed in $L \times L$;

(b) $L$ is a Hausdorff space;

(c) the mapping $\ominus: \leq \rightarrow L$ is continuous.

Proof. For (a), notice that $a \leq b$ iff $a \perp b^{\prime}$. Thus, $\leq=f^{-1}(\perp)$ where $f: L \times L \rightarrow$ $L \times L$ is the continuous mapping $f(a, b)=\left(a, b^{\prime}\right)$. Since $\perp$ is closed, so is $\leq$. That $L$ is Hausdorff now follows by standard arguments (cf. [9, Ch. VII] or [12]). Finally, since $b \ominus a=\left(b^{\prime} \oplus a\right)^{\prime}$, and $\oplus$ and ' are both continuous, $\ominus$ is also continuous.

3.1. Examples. Any product of discrete orthoalgebras, with the product topology, is a TOA. Another source of examples are topological orthomodular lattices (TOMLs) [3, 4. A TOML is an orthomodular lattice equipped with a Hausdorff topology making both the lattice operations and the orthocomplementation continuous. If $L$ is a TOML and $a, b \in L$, then $a \perp b$ iff $a \leq b^{\prime}$ iff $a=a \wedge b^{\prime}$. This is obviously a closed relation, since $L$ is Hausdorff and both $\wedge$ and ' are continuous. Thus, every TOML may be regarded as a TOA. However, there are simple and important examples of lattice-ordered TOAs that are not TOMLs:

Example 3.3. Let $L$ be the horizontal sum of four-element Boolean algebas $L_{x}=$ $\left\{0, x, x^{\prime}, 1\right\}$ with $x$ (and hence, $x^{\prime}$ ) parametrized by a non-degenerate real interval $[a, b]$. Topologize this as two disjoint copies of $[a, b]$ plus two isolated points 0 and 1 . The orthogonality relation is then obviously closed, and $\oplus$ is obviously continuous; however, if we let $x \rightarrow x_{o}$ (with $\left.x \neq x_{o}\right)$ in $I$, then we have $x \wedge x_{o}=0$, yet $x_{o} \wedge x_{o}=x_{o}$; hence, $\wedge$ is not continuous.

Example 3.4. Let $\mathbf{H}$ be a Hilbert space, and let $L=L(\mathbf{H})$ be the space of projection operators on $\mathbf{H}$, with its operator-norm topology. As multiplication is jointly continuous, the relation $P \perp Q$ iff $P Q=Q P=0$ is closed. Since addition and subtraction are continuous, the partial operation $P, Q \mapsto P \oplus Q:=P+Q$ is continuous on $\perp$, as is the operation $P \mapsto P^{\prime}:=\mathbf{1}-P$. Thus, $L(\mathbf{H})$ is a latticeordered topological orthoalgebra. It is not, however, a topological lattice. Indeed, if $Q$ is a non-trivial projection, choose unit vectors $x_{n}$ not lying in $\operatorname{ran}(Q)$, but converging to a unit vector in $x \in \operatorname{ran}(Q)$. If $P_{n}$ is the projection generated by $x_{n}$ and $P$, that generated by $x$, then $P_{n} \rightarrow P$. But $P_{n} \wedge Q=0$, while $P \wedge Q=P$.

We can also endow $L(\mathbf{H})$ with the relative strong operator topology, or, equivalently, the relative weak operator topology (these coinciding for projections). Multiplication is jointly SOT-continuous for operators of norm $\leq 1$, and the same arguments as given above show that this topology also makes $L(\mathbf{H})$ a lattice-ordered TOA, but not a topological lattice.

Remark 3.5. Topologically, projection lattices and TOMLs are strikingly different. Any compact TOML is totally disconnected [4, Lemma 3]. In strong contrast to this, if $\mathbf{H}$ is finite dimensional, then $L(\mathbf{H})$ is compact, but the set of projections of a given dimension in $L(\mathbf{H})$ is a manifold. As this illustrates, TOAs are much freer objects topologically than TOMLs. Indeed, by an easy generalization of Example 3.3, any Hausdorff space can be embedded in a TOA.

3.2. Compact orthoalgebras. For the balance of this paper, we concentrate on compact TOAs. It is a standard fact [9, Corollary VII.1.3] that any ordered topological space with a closed order is isomorphic to a closed subspace of a cartesian power of the real unit interval $[0,1]$ in its product order and topology. It follows 
that such a space $L$ is topologically order-complete, meaning that any upwardlydirected net in $L$ has a supremum to which it converges. Applied to a compact TOA, this yields the following completeness result:

Lemma 3.6. Any compact TOA $L$ is orthocomplete. Moreover, if $A \subseteq L$ is jointly orthogonal, the net of finite partial sums of A converges topologically to $\bigoplus A$.

We are going to show (Theorem 3.12) that any compact regular TOA is atomistic. In aid of this, the following technical definition proves most useful:

Definition 3.7. If $L$ is any orthoalgebra, let

$$
\mathbf{M}(L):=\{(a, c, b) \in L \times L \times L \mid c \leq a, c \leq b, \text { and } a \perp(b \ominus c)\} .
$$

In other words, $(a, c, b) \in \mathbf{M}(L)$ iff $(a \ominus c, c, b \ominus c)$ is a Mackey decomposition for $a$ and $b$.

Lemma 3.8. For any TOA $L$, the relation $\mathbf{M}(L)$ is closed in $L \times L \times L$.

Proof. Just note that $\mathbf{M}(L)=(\geq \times L) \cap(L \times \leq) \cap(\operatorname{Id} \times \ominus)^{-1}(\perp)$. Since the relations $\leq$ and $\perp$ are closed and $\ominus: \leq \rightarrow L$ is continuous, this also is closed.

Since lattice-ordered TOAs need not be topological lattices, the following is noteworthy:

Proposition 3.9. A compact Boolean topological orthoalgebra is a topological lattice, and hence, a compact topological Boolean algebra.

Proof. If $L$ is Boolean, then $\mathbf{M}(L)$ is, up to a permutation, the graph of the mapping $a, b \mapsto a \wedge b$. Thus, by Lemma 3.8, $\wedge$ has a closed graph. Since $L$ is compact, this suffices to show that $\wedge$ is continuous 3 It now follows from the continuity of ' that $\checkmark$ is also continuous.

Note that every compact topological Boolean algebra has the form $2^{E}$, where $E$ is a set and $2^{E}$ has the product topology $[8$. In particular, every compact Boolean algebra is atomistic. This will be useful below.

Question 3.10. Is every Boolean TOA a topological Boolean algebra?

For any orthoalgebra $L$, let $\operatorname{Comp}(L)$ be the set of all compatible pairs in $L$, and for any fixed $a \in L$, let $\operatorname{Comp}(a)$ be the set of elements compatible with $a$.

Proposition 3.11. Let $L$ be a compact TOA. Then

(a) $\operatorname{Comp}(L)$ is closed in $L \times L$;

(b) for every $a \in L, \operatorname{Comp}(a)$ is closed in $L$;

(c) the closure of a pairwise compatible set in $L$ is pairwise compatible;

(d) a maximal pairwise compatible set in $L$ is closed.

Proof. (a) $\operatorname{Comp}(L)=\left(\pi_{1} \times \pi_{3}\right)(\mathbf{M}(L))$. Since $\mathbf{M}(L)$ is closed, and hence compact, and $\pi_{1} \times \pi_{3}$ is continuous, $\operatorname{Comp}(L)$ is also compact, hence closed. For (b), note that $\operatorname{Comp}(a)=\pi_{1}(\operatorname{Comp}(L) \cap(L \times\{a\}))$. As $\operatorname{Comp}(L)$ is closed, so is $\operatorname{Comp}(L) \cap$ $(L \times\{a\})$; hence, its image under $\pi_{1}$ is also closed (remembering here that $L$ is

\footnotetext{
${ }^{3}$ Recall here that if $X$ and $Y$ are compact and the graph $G_{f}$ of $f: X \rightarrow Y$ is closed, then $f$ is continuous. Indeed, let $F \subseteq Y$ be closed. Then $f^{-1}(F)=\pi_{1}\left((X \times F) \cap G_{f}\right)$, where $\pi_{1}$ is projection on the first factor. Since $X$ and $Y$ are compact, $\pi_{1}$ sends closed sets to closed sets.
} 
compact). For (c), suppose $M \subseteq L$ is pairwise compatible. Then $M \times M \subset$ $\operatorname{Comp}(L)$. By part (a), $\operatorname{Comp}(L)$ is closed, so we have

$$
\bar{M} \times \bar{M} \subseteq \overline{M \times M} \subseteq \operatorname{Comp}(L),
$$

whence $\bar{M}$ is again pairwise compatible. Finally, for (d), if $M$ is a maximal pairwise compatible set, then the fact that $M \subseteq \bar{M}$ and $\bar{M}$ is also pairwise compatible entails that $M=\bar{M}$.

There exist (non-orthocoherent) orthoalgebras in which $\operatorname{Comp}(L)=L \times L$ ([5], Example 3.5). However, in an $\mathrm{OML}, \operatorname{Comp}(L)=\mathbf{C}(L)$, the center of $L$. Thus we recover from part (a) of Proposition 3.11 the fact (not hard to prove directly; see 3]) that the center of a compact TOML is a compact Boolean algebra.

In fact, we get a good deal more than this. Recall that an orthoalgebra is regular iff every pairwise compatible subset is contained in a Boolean sub-orthoalgebra. Many orthoalgebras that arise in practice, including all lattice-ordered orthoalgebras, are regular. A block in an orthoalgebra is a maximal Boolean sub-orthoalgebra. In a regular orthoalgebra, this is the same thing as a maximal pairwise compatible set.

Theorem 3.12. Let $L$ be a compact, regular TOA. Then

(a) every block of $L$ is a compact Boolean algebra, as is the center of $L$;

(b) $L$ is atomistic.

Proof. (a) If $L$ is regular, then a block of $L$ is the same thing as a maximal pairwise compatible set. It follows from part (d) of Proposition 3.11 that every block is closed in $L$, and hence compact. It is not hard to show that in a regular TOA the center is the intersection of the blocks. Thus we also have that $\mathbf{C}(L)$ is closed, hence compact. Proposition 3.9 now supplies the result.

To prove (b), suppose $a \in L$. By Zorn's Lemma, there is some block $B \subseteq L$ with $a \in B$. Since $B$ is a compact Boolean algebra, it is complete and atomistic; hence, $a$ can be written as the join, $\bigvee_{B} A$, of a set $A$ of atoms in $B$. Equivalently, $a=\bigvee_{B}\{\bigoplus F \mid F \subseteq A, F$ finite $\}$. By Lemma 3.6, $L$ is orthocomplete; hence, $\bigoplus A=$ $\bigvee_{L}\{\bigoplus F \mid F \subseteq A, F$ finite $\}$ also exists and is the limit of the partial sums $\bigoplus F$, $F \subseteq A$ finite. Since each partial sum lies in $B$, and $B$ is closed, $\bigoplus A \in B$. It follows that $\bigoplus A=a$. It remains to show that every atom of $B$ is an atom of $L$. Suppose that $b$ is an atom of $B$ and that $x \in L$ with $0<x \leq b$. Since $B$ is Boolean, every $y \in B$ satisfies either $b \leq y$ or $y \leq b^{\prime}$; thus, either $x \leq y$ or $y \leq x$. In particular, $x$ is compatible with every element of $B$. Since a block in a regular orthoalgebra is a maximal pairwise compatible set, $x \in B$, whence, $x=b$.

3.3. TOAs with isolated zero. In [3], it is established that any TOML with an isolated point is discrete. In particular, a compact TOML with an isolated point is finite. This does not hold for lattice-ordered TOAs generally. Indeed, if $\mathbf{H}$ is a finite-dimensional Hilbert space, then $L(\mathbf{H})$ is a compact lattice-ordered TOA in which 0 is isolated. On the other hand, as we now show, a compact TOA with isolated zero does have quite special properties. We begin with an elementary but important observation. Call an open set in a TOA space totally non-orthogonal if it contains no two orthogonal elements.

Proposition 3.13. Every non-zero element of a TOA has a totally non-orthogonal open neighborhood. 
Proof. Let $L$ be a TOA. If $a \neq 0$, then $(a, a) \notin \perp$. Since the latter is closed in $L^{2}$, we can find open sets $U$ and $V$ with $(a, a) \in U \times V$ and $(U \times V) \cap \perp=\emptyset$. The set $U \cap V$ is a totally non-orthogonal open neighborhood of $a$.

Proposition 3.14. Let $L$ be a compact TOA with 0 isolated. Then

(a) $L$ is atomistic and of finite height;

(b) the set of atoms of $L$ is open.

Proof. (a) We first show that there is a finite upper bound on the size of a pairwise orthogonal set. Since 0 is isolated in $L, L \backslash\{0\}$ is compact. By Lemma 3.13, we can cover $L \backslash\{0\}$ by finitely many totally non-orthogonal open sets $U_{1}, \ldots, U_{n}$. A pairwise-orthogonal subset of $L \backslash\{0\}$ can meet each $U_{i}$ at most once, and so, can have at most $n$ elements. Now given a finite chain $x_{1}<x_{2}<\ldots<x_{m}$ in $L$, we can construct a pairwise orthogonal set $y_{1}, \ldots, y_{m-1}$ defined by $y_{1}=x_{1}$ and $y_{k}=x_{k} \ominus y_{k-1}$ for $k=2, \ldots, m-1$. Hence, $m-1 \leq n$, so $m \leq n+1$. This shows that $L$ has finite height, from which it follows that $L$ is atomistic.

(b) Note that if $A$ and $B$ are any closed subsets of $L$, then $(A \times B) \cap \perp$ is a closed, hence compact, subset of $\perp$. Since $\oplus$ is continuous on $\perp$, the set

$$
A \oplus B:=\{a \oplus b \mid a \in A, b \in B \text { and } a \perp b\}=\bigoplus((A \times B) \cap \perp)
$$

is compact, hence closed. The set of non-atoms is precisely $(L \backslash\{0\}) \oplus(L \backslash\{0\})$. Since 0 is isolated, $(L \backslash\{0\})$ is closed. Thus, the set of non-atoms is closed.

Remark 3.15. Notice that both the statements and the proofs of Lemma 3.13 and part (a) of Proposition 3.14 apply verbatim to any topological orthoposet, i.e., any ordered space having a closed order and equipped with a continuous orthocomplementation.

If $a$ belongs to the center of a TOA $L$, then $[0, a] \times\left[0, a^{\prime}\right] \subseteq \perp$. Hence, the natural isomorphism $\phi:[0, a] \times\left[0, a^{\prime}\right] \rightarrow L$ given by $(x, y) \mapsto x \oplus y$ is continuous. If $L$ is compact, then so are $[0, a]$ and $\left[0, a^{\prime}\right]$; hence, $\phi$ is also a homeomorphism. Since the center of an orthoalgebra is a Boolean sub-orthoalgebra of $L$, and since a Boolean algebra of finite height is finite, Proposition 3.14 has the following

Corollary 3.16. Let $L$ be a compact TOA with 0 isolated. Then the center of $L$ is finite. In particular, $L$ decomposes, both algebraically and topologically, as the product of finitely many compact irreducible TOAs.

\section{Stably ordered topological orthoalgebras}

In this section we consider a particularly tractable, but still quite broad, class of TOAs.

Definition 4.1. We shall call an ordered topological space $L$ stably ordered iff, for every open set $U \subseteq L$, the upper-set $U \uparrow=\{b \in L \mid \exists a \in U, a \leq b\}$ is again open 4

Remark 4.2. Note that this is equivalent to saying that the second projection mapping $\pi_{2}: \leq \rightarrow L$ is an open mapping, since for open sets $U, V \subseteq L$,

$$
\pi_{2}((U \times V) \cap \leq)=U \uparrow \cap V .
$$

Note, too, that if $L$ carries a continuous orthocomplementation ', then $L$ is stably ordered iff $U \downarrow=\{x \mid \exists y \in U, x \leq y\}$ is open for all open sets $U \subseteq L$.

\footnotetext{
${ }^{4}$ The term used by Priestley 13 is "space of type $I_{i}$ ".
} 
Example 4.3. The following example (a variant of Example 3.3) shows that a TOA need not be stably ordered. Let $L=[0,1 / 4] \cup[3 / 4,1]$ with its usual topology, but without its usual order. For $x, y \in L$, set $x \perp y$ iff $x+y=1$ or $x=0$ or $y=0$. In any of these cases, define $x \oplus y=x+y$. As is easily checked, this is a compact lattice-ordered TOA. However, for the clopen set $[0,1 / 4]$ we have $[0,1 / 4] \uparrow=[0,1 / 4] \cup\{1\}$, which is certainly not open.

Such examples notwithstanding, most of the orthoalgebras that arise "in nature" do seem to be stably ordered. The following is mentioned (without proof) in [13]:

Lemma 4.4. Any topological $\wedge$-semilattice - in particular, any topological lattice - is stably ordered.

Proof. If $L$ is a topological meet-semilattice and $U \subseteq L$ is open, then

$$
U \uparrow=\{x \in L \mid \exists y \in U x \wedge y \in U\}=\pi_{1}\left(\wedge^{-1}(U)\right)
$$

where $\pi_{1}: L \times L \rightarrow L$ is the (open) projection map on the first factor and $\wedge$ : $L \times L \rightarrow L$ is the (continuous) meet operation.

Many orthoalgebras, including projection lattices, can be embedded in ordered abelian groups [1. Indeed, suppose $G$ is an ordered abelian group. If $e>0$ in $G$, let $[0, e]$ denote the set of all elements $x \in G$ with $0 \leq x \leq e$. We can endow $[0, e]$ with the following partial-algebraic structure: for $x, y \in[0, e]$, set $x \perp y$ iff $x+y \leq e$, in which case let $x \oplus y=x+y$. Define $x^{\prime}=e-x$. Then $\left([0, e], \oplus,{ }^{\prime}, 0, e\right)$ is an effect algebra. By a faithful sub-effect algebra of $[0, e]$, we mean a subset $L$ of $[0, e]$, containing 0 and $e$, closed under both $\oplus$, where this is defined, and under ', and such that, for all $x, y \in L, x \leq y$ in $G$ iff $\exists z \in L$ with $y=x+z$ (so that the order inherited from $G$ and the order induced by $\oplus$ coincide).

By way of example, let $L=L(\mathbf{H})$, the projection lattice of a Hilbert space $\mathbf{H}$, regarded as an orthoalgebra, and let $G=\mathcal{B}_{s a}(\mathbf{H})$, the ring of bounded self-adjoint operators on $\mathbf{H}$ with its operator-norm topology, ordered in the usual way. Then $L$ is a faithful sub-effect algebra of $[0, \mathbf{1}]$, where $\mathbf{1}$ is the identity operator on $\mathbf{H}$. (This follows from the fact that, for projections $P, Q \in L(\mathbf{H}), P+Q \leq \mathbf{1}$ iff $P \perp Q$, and the fact that if $P \leq Q$ as positive operators, then $Q-P$ is a projection.)

Lemma 4.5. Let $L$ be an orthoalgebra, let $G$ be any ordered topological abelian group with a closed cone (equivalently, a closed order), and suppose that $L$ can be embedded as a sub-effect algebra of $[0, e]$, where $e>0$ in $G$. Then $L$, in the topology inherited from $G$, is a stably ordered TOA.

Proof. We may assume that $L$ is a subspace of $[0, e]$. Since $x \perp y$ in $L$ iff $x+y \leq e$, we have $\perp=+^{-1}([0, e]) \cap L$, which is relatively closed in $L$. The continuity of $\oplus$ and ' are automatic. Suppose now that $U \cap L$ is a relatively open subset of $L$. Then, since $L$ is a faithful sub-effect algebra of $[0, e]$, the upper set generated by $U \cap L$ in $L$ is $U \uparrow \cap L$, where $U \uparrow$ is the upper set of $U$ in $[0, e]$. It suffices to show that this last is open. But $U \uparrow=\bigcup_{y \geq 0} U+y$, which is certainly open.

In particular, it follows that the projection lattice $L(\mathbf{H})$ of a Hilbert space $\mathbf{H}$ is stably ordered in its norm topology.

Example 4.6. A state on an orthoalgebra $(L, \oplus)$ is a mapping $f: L \rightarrow[0,1]$ such that $f(1)=1$ and, for all $a, b \in L, f(a \oplus b)=f(a) \oplus f(b)$ whenever $a \oplus b$ exists. A set $\Delta$ of states on $L$ is said to be order-determining iff $f(p) \leq f(q)$ for all $f \in \Delta$ 
implies $p \leq q$ in $L$. In this case the mapping $L \rightarrow \mathbb{R}^{\Delta}$ given by $p \mapsto \hat{p}$, where $\hat{p}(f)=f(p)$, embeds $L$ as a faithful sub-effect algebra of the ordered abelian group $\mathbb{R}^{\Delta}$ (with pointwise addition, pointwise order). Since the positive cone of $\mathbb{R}^{\Delta}$ is closed in the product topology, Lemma 4.5 tells us that $L$ is stably ordered in the topology inherited from pointwise convergence on $\Delta$.

As a special case, the projection lattice $L=L(\mathbf{H})$ has an order-determining set of states of the form $f(P)=\langle P x, x\rangle$, where $x$ is a unit vector in $\mathbf{H}$. If $\mathbf{H}$ is complex, the polarization identity tells us that the weakest topology on $L(\mathbf{H})$ making every such state continuous is exactly the restriction to $L(\mathbf{H})$ of the weak operator topology. Recall that this coincides with the strong operator topology on $L(\mathbf{H})$, and makes $L(\mathbf{H})$ a TOA. Thus, for a complex Hilbert space, $L(\mathbf{H})$ is stably-ordered in its strong operator topology.

If $U$ and $V$ are subsets of an orthoalgebra $L$, let us write $U \oplus V$ for $\bigoplus((U \times V) \cap \perp)$, i.e., for the set of all (existing) orthogonal sums $a \oplus b$ with $a \in U$ and $b \in V$.

Lemma 4.7. A TOA L is stably ordered if, and only if, for every pair of open sets $U, V \subseteq L$, the set $U \oplus V$ is also open.

Proof. Let $U$ and $V$ be any two open sets in $L$. Then

$$
\begin{aligned}
U \oplus V & =\{c \in L \mid c=a \oplus b, a \in U, b \in V\} \\
& =\{c \in L \mid \exists a \in U a \leq c \text { and } c \ominus a \in V\} \\
& =\pi_{2}\left(\ominus^{-1}(V) \cap(L \times U \uparrow)\right) .
\end{aligned}
$$

Now, since $L$ is stably ordered, $U \uparrow$ is open, and hence, $\ominus^{-1}(V) \cap(L \times U \uparrow)$ is relatively open in $\leq$. But as observed above, for $L$ stably ordered, $\pi_{2}: \leq \rightarrow L$ is an open mapping, so $U \oplus V$ is open. For the converse, just note that $U \uparrow=U \oplus L$.

Proposition 3.12 tells us that a compact TOA $L$ with 0 isolated is atomistic and of finite height. It follows easily that every element of $L$ can be expressed as a finite orthogonal sum of atoms. Let the dimension, $\operatorname{dim}(a)$, of an element $a \in L$ be the minimum number $n$ of atoms $x_{1}, \ldots, x_{n}$ such that $a=x_{1} \oplus \cdots \oplus x_{n}$. Note that $a \in L$ is an atom iff $\operatorname{dim}(a)=1$.

Theorem 4.8. Let $L$ be a compact, stably-ordered TOA in which 0 is an isolated point. Then

(a) the set of elements of $L$ of a given dimension is clopen;

(b) the topology on $L$ is completely determined by that on the set of atoms.

Proof. We begin by noting that if $A$ and $B$ are clopen subsets of $L$, then $A \oplus B$ is again clopen (open, by Lemma 4.7, and closed, because the image of the compact set $(A \times B) \cap \perp$ under the continuous map $\oplus)$. Now, since 0 is isolated, $L \backslash\{0\}$ is clopen. Since the set of non-atoms in $L$ is exactly $(L \backslash\{0\}) \oplus(L \backslash\{0\})$, it follows that the set of atoms is clopen. Define a sequence of sets $L_{k}, k \in \mathbb{N}$, by setting $L_{0}=\{0\}$, $L_{1}=$ the set of atoms of $L$, and $L_{k+1}:=L_{k} \oplus L_{1}$. These sets are clopen, as are all Boolean combinations of them. Thus, $\{a \in L \mid \operatorname{dim}(a)=k\}=L_{k} \backslash\left(\bigcup_{i=0}^{k-1} L_{k}\right)$ is clopen for every $k=0, \ldots, \operatorname{dim}(L)$. This proves (a). For (b), it now suffices to show that the topology on each $L_{k}$ is determined by that on $L_{1}$. Since $L_{1}$ and $L_{2}$ are clopen, Lemma 4.7 tells us that the mapping $\oplus:\left(\left(L_{k} \times L_{1}\right) \cap \perp\right) \rightarrow L_{k+1}$ is an open surjection, and hence, a quotient mapping. Thus, the topology on $L_{k+1}$ is entirely determined by that on $L_{k}$ and that on $L_{1}$. 


\section{REFERENCES}

[1] Bennett, M. K., and Foulis, D. J., Interval effect algebras and unsharp quantum logics, Advances in Applied Math. 19 (1997), 200-219. MR.1459498(98m:06024)

[2] Bruns, G., and Harding, J., The algebraic theory of orthomodular lattices, in B. Coecke, D. J. Moore and A. Wilce (eds.), Current Research in Operational Quantum Logic, Kluwer: Dordrecht (2000). MR1907155

[3] Choe, T. H., and Greechie, R. J., Profinite Orthomodular Lattices, Proc. Amer. Math. Soc. 118 (1993), 1053-1060. MR.1143016 (93j:06008)

[4] Choe, T. H., Greechie, R. J., and Chae, Y., Representations of locally compact orthomodular lattices, Topology and its Applications 56 (1994) 165-173. MR1266141 (95c:06017)

[5] Foulis, D. J., Greechie, R. J., and Ruttimann, G. T., Filters and Supports on Orthoalgebras Int. J. Theor. Phys. 31 (1992) 789-807. MR.1162623 (93c:06014)

[6] Foulis, D. J., and Randall, C. H., What are quantum logics, and what ought they to be?, in Beltrametti, E., and van Fraassen, B. C. (eds.), Current Issues in Quantum Logic, Plenum: New York, 1981.) MR0723148 (84k:03142)

[7] Greechie, R. J., Foulis, D. J., and Pulmannová, S., The center of an effect algebra, Order 12 (1995), 91-106. MR 1336539(96c:81026)

[8] Johnstone, P. T., Stone Spaces, Cambridge: Cambridge University Press, 1982. MR0698074 (85f:54002)

[9] Kalmbach, G., Orthomodular Lattices, Academic Press, 1983. MR0716496 (85f:06012)

[10] Nachbin, L., Topology and Order, van Nostrand: Princeton 1965. MR0219042 (36:2125)

[11] Priestley, H. A., Ordered Topological Spaces and the Representation of Distributive Lattices, Proc. London Math. Soc. 24 (1972), 507-530. MR0300949 (46:109)

[12] Pulmannová, S., and Riečanova, Z., Block-finite Orthomodular Lattices, J. Pure and Applied Algebra 89 (1993), 295-304. MR 1242723 (94j:06016)

[13] Riečanova, Z., Order-Topological Lattice Effect Algebras, preprint, 2003.

[14] Wilce, A., Test Spaces and Orthoalgebras, in Coecke et al (eds.) Current Research in Operational Quantum Logic, Kluwer: Dordrecht (2000). MR1907157|(2003c:81018)

Department of Mathematics, Susquehanna University, Selinsgrove, Pennsylvania 17870

E-mail address: wilce@susqu.edu 